\title{
Teaching Method of Base Body Creation in Engineering Graphics Course combination with UG NX
}

\author{
Xiaoda Li ${ }^{\mathrm{a},{ }^{\star},}$, Xianghui Zhan ${ }^{\mathrm{b}}$ \\ Zhuhai College of Jilin University, Zhuhai, China \\ aliliabc0185@sina.com, bzhanxh_1215@163.com \\ ${ }^{*}$ Corresponding author
}

Keywords: Basic body; Engineering graphics; NX; Teaching method

\begin{abstract}
It discusses the basic knowledge and generating method of Base Body in the course of traditional Engineering Graphics, and produces the teaching methods about the creating of Basic body in the course combined with NX software in the paper. Solid model can be divided into base body and assembly. The simple base body can be gotten by using Primitive Feature of NX, and the more complex assembly model can be implemented by sweeping the sketch of NX using some sweeping features like Extrude and Rotate etc. The practice has proved that the method about the combination of $\mathrm{NX}$ and the traditional theory could make the knowledge points more intuitive and easier in teaching, and could also improve the efficiency and teaching quality.
\end{abstract}

\section{The concept of base body}

According the complexity of the model, it can be divided into Base Body and Assembly [1-2].The basic geometry with the simple shape is called Base Body. According the difference of the surface's geometry shape, it is divided into two categories including Planar Object and Curved Surface Object. The 3D (three dimensional) geometry with all flat surfaces is called Planar Object, such as Prism, Pyramid etc. as shown in Fig. 1. As shown in Fig. 2, the geometry with all curved surface, and the surface made up by curved surfaces and flat surfaces is called curved surface object, such as Cylinders, Cones, Spheres and Rings etc.
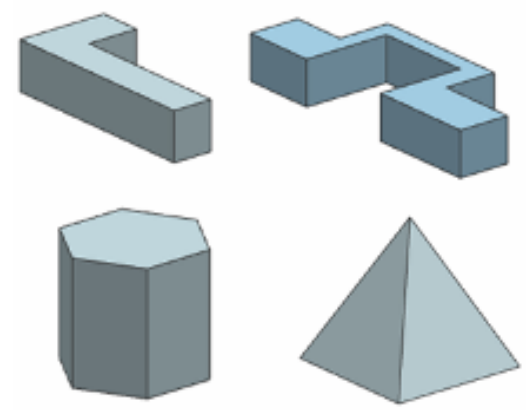

Figure. 1 The planar objects

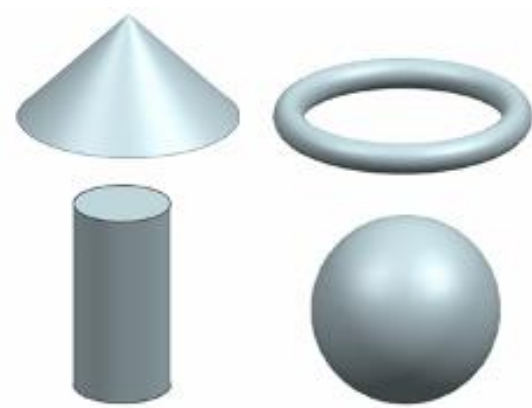

Figure. 2 The curved surface objects

\section{The configuration of Base Body in the conventional mechanical drawing}

As shown in Fig. 3 and Fig. 4, usually the base body can be gotten by rotating or moving sectional geometry objects, which can be lines and surfaces etc. 


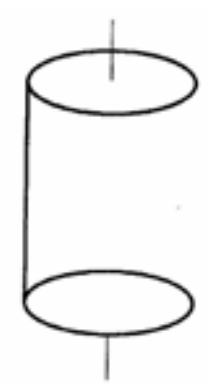

Figure. 3 The base body formed by rotating
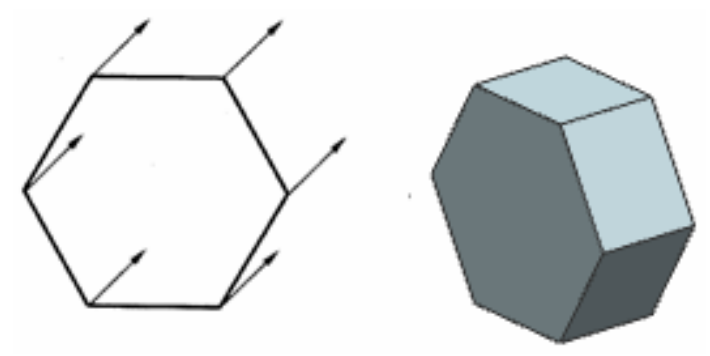

Figure. 4 The base body formed by moving

\section{The fundamental concepts creating base body with NX}

NX's modeling process based on the feature is simulated the real machining process of parts, firstly build the blank, and then have the roughing, at last finish precision work. The first step 'building blank' of NX's Solid Modeling can be considered to create the base body of a model [3-4]. There are two tools creating the blank feature: Creating the basic blank solid with Primitive Feature like Rectangular, Cylinder, Cone and Sphere and with Swept Feature such as Extrude, Revolve, and Sweep along the guide line and Tube [5-6].

\section{The implementation of base body combined with NX}

Primitive Feature. The forming of Cylinder. Cylindrical is surrounded by top round, bottom round and cylindrical surface. As shown in Fig. 5, the cylindrical surface can be gotten through rotating around the rotary axis ' $\mathrm{OO} 1$ ' for 360 degrees with the line 'AA1' parallelling to the rotary axis. In software NX, click the command 'Insert' $\rightarrow$ 'Design Feature' $\rightarrow$ 'Cylinder', as shown in Fig. 6, and then create the cylinder with the direction vector, diameter and height.

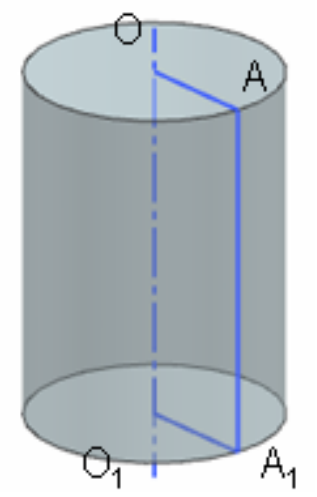

Figure. 5 The cylinder

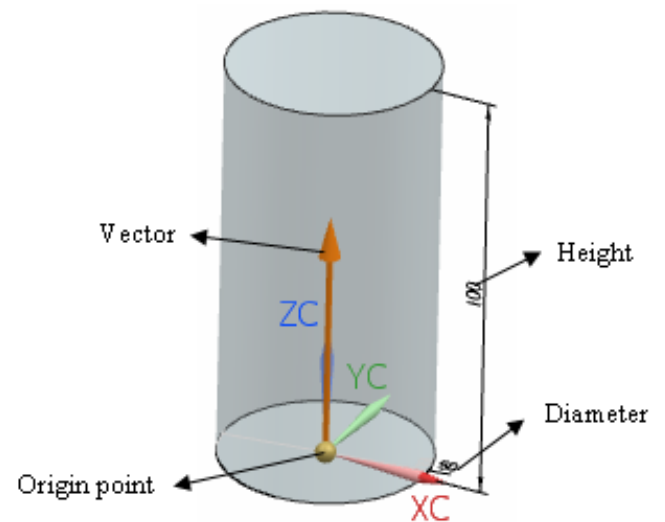

Figure. 6 The cylinder created by NX

The forming of Cone. Cone is surrounded by bottom round and conical surface. As shown in Fig. 7, the conical surface can be gotten through rotating around the rotary axis 'OO1' for 360 degrees with the generatrix 'SA'(intersect with the rotary axis). Click the command 'Insert' $\rightarrow$ 'Design Feature' $\rightarrow$ 'Cone', and it can be created the basic cone and frustum solid. Fig. 8 shows the meaning of the parameters involved. 


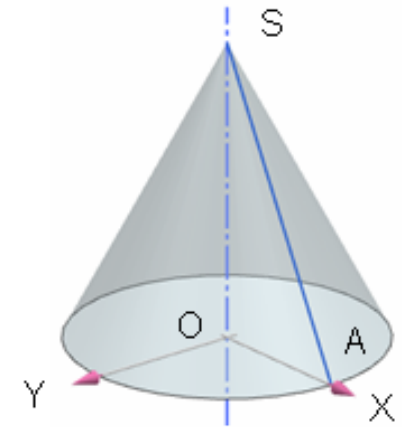

Figure. 7 The cone

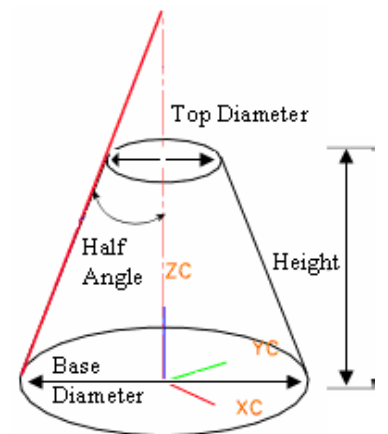

Figure. 8 The meaning of the cone's parameters

The forming of Sphere. As shown in Fig. 9, the sphere can be gotten through rotating around the rotary axis ' $\mathrm{OO} 1$ ' for 360 degrees with the circle for the generatrix. In software NX, click the command 'Insert' $\rightarrow$ 'Design Feature' $\rightarrow$ 'Sphere', and it can be created the sphere solid according the actual needs for two kinds of method: 'Center and Diameter' and 'Arc'.

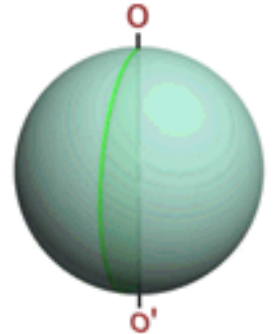

Figure. 9 The sphere

Achieving the creation of basic body with the Sketch of NX . Basic knowledge about the sketch. The sketch is a collection of a set of two-dimensional curves and points on the sketch plane, and is a reusable object. It can build some new features with the sketch. The main functions of sketches include: Controlling the design contour and section, being generated solids and sheet bodies by sweeping, extruding and rotating sketchs, creating the large-scale and two-dimensional concept layout by using the sketch curve, and building construction geometry, such as motion path, arc gap etc.

The most important contents of a sketch include: selecting the sketch plane, adding sketch curves, adding geometric constraints and dimensional constraints for the sketch's line string, editting the sketch, and the subsequent feature operating of the sketch. It can select the sketch curve to generate base body by using commands like Sweep, Extrude, and Tube etc.

The sweeping method of translating and extruding. The translating method is to bulid the base body by translating any plane graphics (basal plane) for some distance along a straight line direction. As shown in Fig. 10, the base body formed by translating the hexagon for some distance along the specified direction is called Prism, which is composed by the upper and lower undersides and several ribs. Hexagon can be drawn by using the sketch of NX and the prism solid can be generated by using Extrude feature.

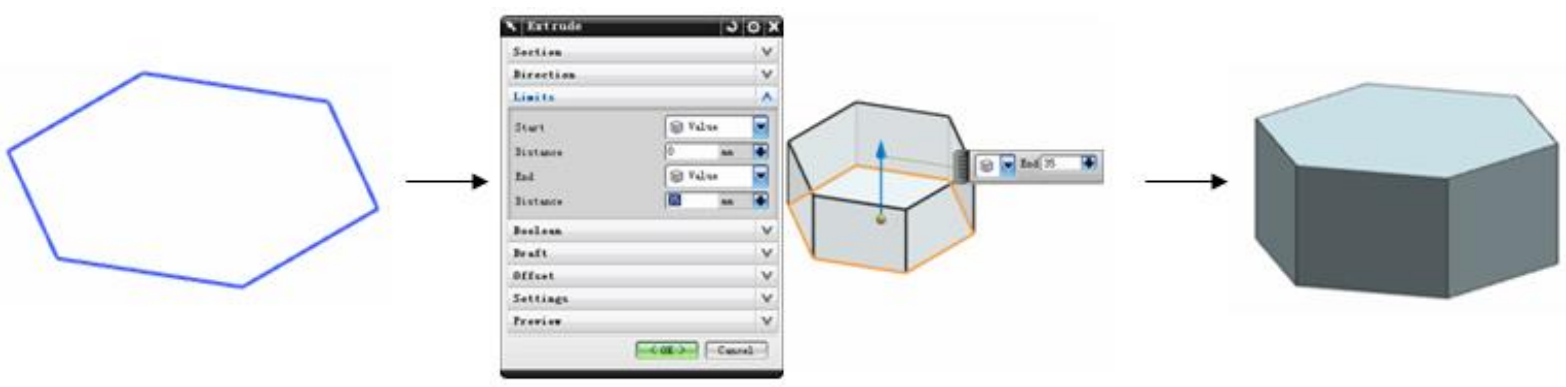

Figure. 10 The prism 
The sweeping method of rotating. The sweeping method of rotating is to build a combined rotator by rotating the section curve for some angel along the specified axis. As is shown in Fig. 11, it needs to specify the rotaing section curves, rotating center axis, rotating angel to use Sweep feature, and at last generate the base solid of rotating.
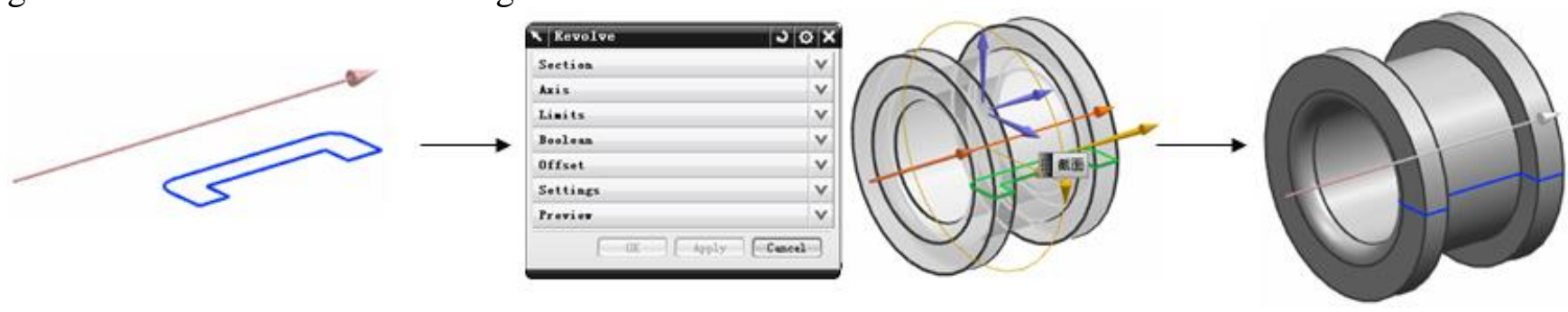

Figure. 11 The generated combined rotater with the rotating mether

The base body generated by using oriented method. Create the base body through selecting the existing surface of body to sweep along the guide lines. As shown in Fig. 12, it needs to select section line and guide line to have the sweeping operation along the guide line.
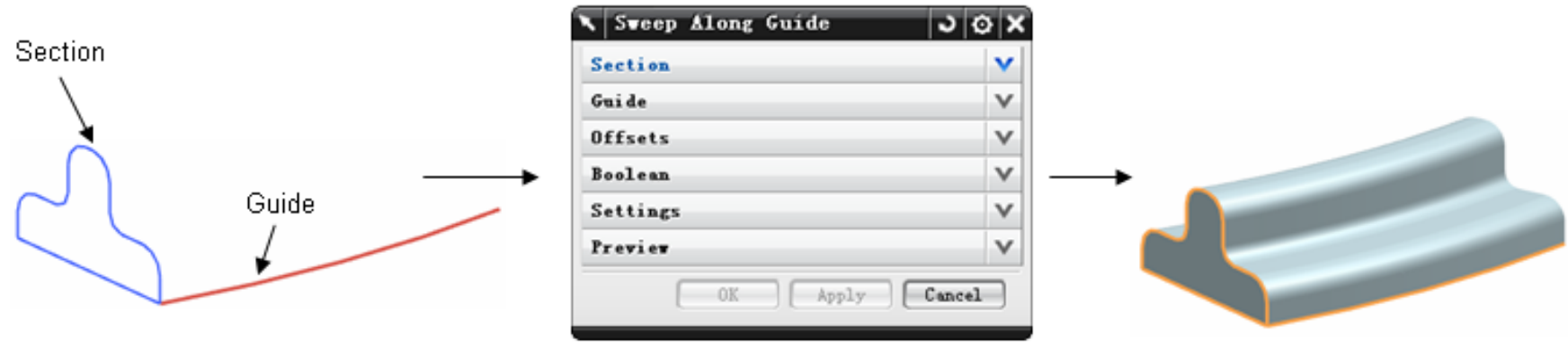

Figure. 12 The base body swept by the plane curve

\section{Summary}

The software 'Siemens NX' is widely used in industrial areas such as mechanical, electronics, etc. And it can improve the speed and efficiency of design [7-8]. It is a direction of teaching reform introducing the 3D design to the traditional theory of teaching [9-10]. In the paper, it discusses the combination of the knowledge point on the base body of Engineering Graphics with NX, and the research can be extended to all the knowledge in different courses.

\section{Acknowledgements}

It is highly appreciated that Siemens PLM software provided the NX software. This work is supported by a grant from the Teaching Quality Project (ZLGC20100208; ZLGC20140605; PY2014008) and the 'Three Levels' backbone teacher training program of Zhuhai College of Jilin University.

\section{References}

[1] H.S. HOU: Mechanical Engineering Graphics (Science Press, Beijing 2007).

[2] M.X. HE: Mechanical Engineering Graphics (High Education Press, Beijing 2007).

[3] Siemens PLM Software, NX8.5 Help Library, 2013.

[4] Siemens PLM Software, Computer Assisted Self Teach for NX8.5, 2013.

[5] R.J. HONG: NX4 CAD QuickStarts Guidance (Tsinghua University Press, Beijing 2006). 
[6] R.J. HONG: UG NX6 Synchronous Modeling Technology Training Course (Tsinghua University Press, Beijing 2008).

[7] Li Xiaoda, Zhan xianghui, Study on the Principle of Reasonable Modeling Based on NX, Applied Mechanics and Materials, Vol. 157-158, PP. 684-687, 2012.

[8] Zhan xianghui, Li Xiaoda, The method and Technique of 2D Drawing Creation Based on NX7.5, Applied Mechanics and Materials, Vol. 157-158, PP. 119-122, 2012.

[9] WANG Qian, The application of Pro/E virtual stimulation in teaching of lathe assembling/disassembling training, Manufacturing Technology \& Machine Tool, No.2, pp. 29-31, 2012.

[10] LI Qing-hua, Programming of engineering graphics 3D parts library based on CATIA software, Machinery Design \& Manufacture, No.12, pp. 68-69, 2005. 